\title{
A Nuclear Magnetic Resonance Study of the Molecular Mobility in $\mathrm{Ba}\left(\mathrm{NO}_{2}\right)_{2} \cdot \mathrm{H}_{2} \mathrm{O}$
}

\author{
Karin Larsson* and Jörgen Tegenfeldt
}

Institute of Chemistry, University of Uppsala, Box 531, S-75121 Uppsala, Sweden

\begin{abstract}
Larsson, K. and Tegenfeldt, J., 1987. A Nuclear Magnetic Resonance Study of the Molecular Mobility in $\mathrm{Ba}\left(\mathrm{NO}_{2}\right)_{2} \cdot \mathrm{H}_{2} \mathrm{O}$. - Acta Chem. Scand., Ser. A 41: 59-66.

Diffusive dynamical properties of molecules and ions in the pyro- and piezoelectric compound $\mathrm{Ba}\left(\mathrm{NO}_{2}\right)_{2} \cdot \mathrm{H}_{2} \mathrm{O}$ were investigated by ${ }^{1} \mathrm{H} \mathrm{NMR}$, measuring the spin-lattice relaxation times in the laboratory and rotating frames $\left(T_{1}\right.$ and $\left.T_{1 \mathrm{\rho}}\right)$ and the dipolar relaxation time $\left(T_{1 \mathrm{D}}\right)$. Single crystals were used for measuring these relaxation times as a function of orientation, and polycrystalline samples for measuring temperature dependences. $\mathrm{Ba}\left(\mathrm{NO}_{2}\right)_{2} \cdot \mathrm{H}_{2} \mathrm{O}$ undergoes a reversible phase transition at $350 \mathrm{~K}$, followed by water loss on heating.

In the room temperature phase, two major processes were found to be responsible for the relaxation. These dominate the relaxation in separate temperature regions. The activation energies, $E$, associated with the low and the high temperature regions are $25 \mathrm{~kJ} \mathrm{~mol}^{-1}$ and $57 \mathrm{~kJ} \mathrm{~mol}^{-1}$, respectively. The high activation energy process is clearly a $180^{\circ}$ flip motion of the $\mathrm{H}_{2} \mathrm{O}$ molecules; the other process could not be clearly identified.

The pre-exponential factor in the Arrhenius relation for the correlation times of the $\mathrm{H}_{2} \mathrm{O}$ flip motion is $1 \times 10^{-15} \mathrm{~s}$.
\end{abstract}

This work is part of a series of investigations ${ }^{1}$ in which molecular (especially $\mathrm{H}_{2} \mathrm{O}$ ) and ionic mobility in the solid state is studied by pulsed NMR, with the objective of establishing the particular nature of the diffusive dynamics and the rate constants of the Arrhenius relation.

Both rotational and translational diffusion of water molecules in solids have been studied previously. ${ }^{2}$ The most frequent reorientational motion is a $180^{\circ}$ flip of the molecule about its twofold axis.

The compound $\mathrm{Ba}\left(\mathrm{NO}_{2}\right)_{2} \cdot \mathrm{H}_{2} \mathrm{O}$ has been studied in the present paper. This salt is both pyro- and piezoelectric ${ }^{3}$ and crystallizes at room temperature in the hexagonal system with space group $P 6_{5}$ or its enantiomorph $P 6_{1}{ }^{4,5}$ All $\mathrm{H}_{2} \mathrm{O}$ molecules in the unit cell are crystallographically equivalent and form hydrogen bonds to the two independent $\mathrm{NO}_{2}{ }^{-}$ions. One of the hydrogen bonds of the $\mathrm{H}_{2} \mathrm{O}$ molecule is donated to the nitrogen atom in one of the nitrite ions; the other

\footnotetext{
*To whom correspondence should be addressed

5* Acta Chemica Scandinavica A41 (1987) $\quad 59-66$
}

one is a bifurcated hydrogen bond involving two oxygen atoms in different nitrite ions (see Fig. 1).

$\mathrm{Ba}\left(\mathrm{NO}_{2}\right)_{2} \cdot \mathrm{H}_{2} \mathrm{O}$ undergoes a sluggish, reversible phase transition at about $350 \mathrm{~K}$. Infrared spectroscopic evidence and entropy considerations suggest that above this temperature, the water molecules are orientationally disordered, each water molecule having 5 different available orientations. ${ }^{6,7}$ No details are known, however, concerning the structure of this phase.

\section{Experimental}

Single crystals of $\mathrm{Ba}\left(\mathrm{NO}_{2}\right)_{2} \cdot \mathrm{H}_{2} \mathrm{O}$ were grown from a saturated aqueous solution of $\mathrm{Ba}\left(\mathrm{NO}_{2}\right)_{2} \cdot \mathrm{H}_{2} \mathrm{O}$ (purum p.a.) by slow evaporation at room temperature. For the NMR measurements, two crystals were mounted on teflon pins for rotation about two approximately orthogonal axes with components $(0,0,1)$ (axis 1) and $(-0.083,0.081,0.001)$ (axis 2$)$ with respect to the crystal axes. The orientation was determined using an X-ray diffractometer. 


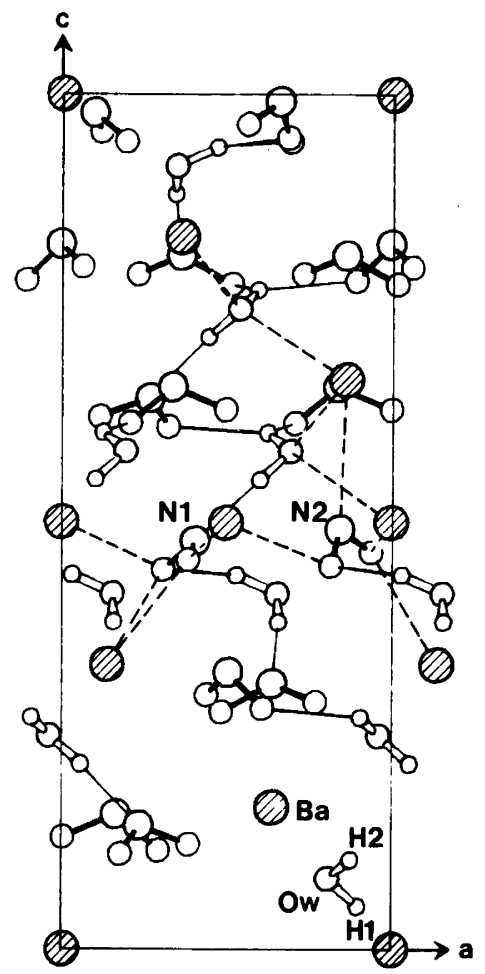

Fig. 1. Crystal structure of $\mathrm{Ba}\left(\mathrm{NO}_{2}\right)_{2} \cdot \mathrm{H}_{2} \mathrm{O}$, room temperature phase.

Coarse-grained samples of this compound, grown from aqueous solutions, have been shown to contain as much as $50 \%$ trapped excess water, whereas finely ground samples approach the stoichiometric composition $\mathrm{Ba}\left(\mathrm{NO}_{2}\right)_{2} \cdot \mathrm{H}_{2} \mathrm{O} .{ }^{6}$ In our measurements on polycrystalline specimens we therefore used finely ground material, and the NMR spectra clearly showed that the material used contained only minor amounts of trapped excess water. Furthermore, all samples were contained in sealed Pyrex tubes during the measurements, to reduce water loss on heating.

The experimental results reported in this paper were obtained by measuring the proton relaxation times $T_{1}, T_{1 \mathrm{e}}$ and $T_{1 \mathrm{D}}$ using a pulsed NMR spectrometer. When measuring the spin-lattice relaxation time in the laboratory frame $\left(T_{1}\right)$ a string of $90^{\circ}$ pulses (saturation pulses) were used, followed by a $90^{\circ}$ detection pulse. The spin-lattice relaxation time in the rotating frame $\left(T_{10}\right)$ was measured using the spin-locking technique, and

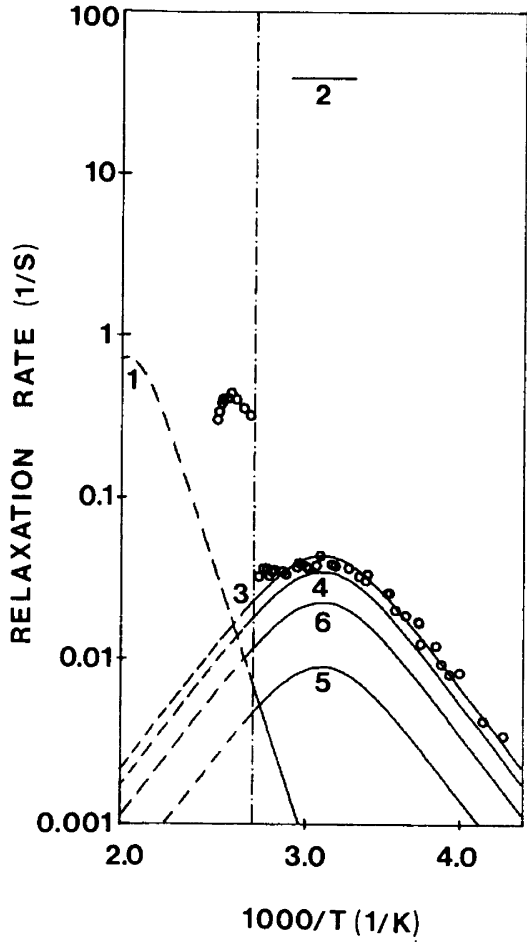

Fig. 2. Temperature dependence of the proton relaxation rate $\left(T_{1}^{-1}\right)$ in polycrystalline $\mathrm{Ba}\left(\mathrm{NO}_{2}\right)_{2} \cdot \mathrm{H}_{2} \mathrm{O}$. Circles are experimental values for $v_{0}=89.4 \mathrm{MHz}$. The calculated rates are indicated by $1-6$ for models $1-6$. The rate maximum is indicated only for model 2 . The rate maxima for models 7 and 8 are $<1 \times 10^{-3} \mathrm{~s}^{-1}$ and are not shown in this figure. The rate based on model 1 was calculated using the parameters $E$ and $\tau_{0}$, obtained from the $T_{10}$ measurements. The vertical dashed line shows the temperature of the phase transition $(350 \mathrm{~K})$. Note that the calculated rates for the high temperature phase in this figure (dashed part of the curves) are based on the room temperature structure and do not, therefore, necessarily represent the true rates.

the dipolar relaxation time $\left(T_{1 \mathrm{D}}\right)$ using the Jeener-Broekaert technique.

Using the powdered sample, $T_{1}$ was measured as a function of temperature in the interval 230$385 \mathrm{~K}$ and at the resonance frequency $\left(v_{0}\right) 89.4$ $\mathrm{MHz} . T_{1}$ was also measured on the single crystals as a function of orientation, both for axis 1 and axis 2. The temperature during these measurements was $300 \mathrm{~K}$, with $v_{0}=89.8 \mathrm{MHz}$. The results of the $T_{1}$ measurements are shown in Figs. 2 and 3 . 
NMR STUDY OF $\mathrm{Ba}\left(\mathrm{NO}_{2}\right)_{2} \cdot \mathrm{H}_{2} \mathrm{O}$

Fig. 3. Orientational dependences of proton $T_{1}^{-1}$ for two single crystals of $\mathrm{Ba}\left(\mathrm{NO}_{2}\right)_{2} \cdot \mathrm{H}_{2} \mathrm{O}$; rotation about (a) axis 1 and (b) axis 2.

Circles are experimental values for $v_{0}=89.8 \mathrm{MHz}$. The calculated rates (corresponding to $T=300 \mathrm{~K}$ ) are indicated by 3,4 and 6 for models 3,4 and 6.
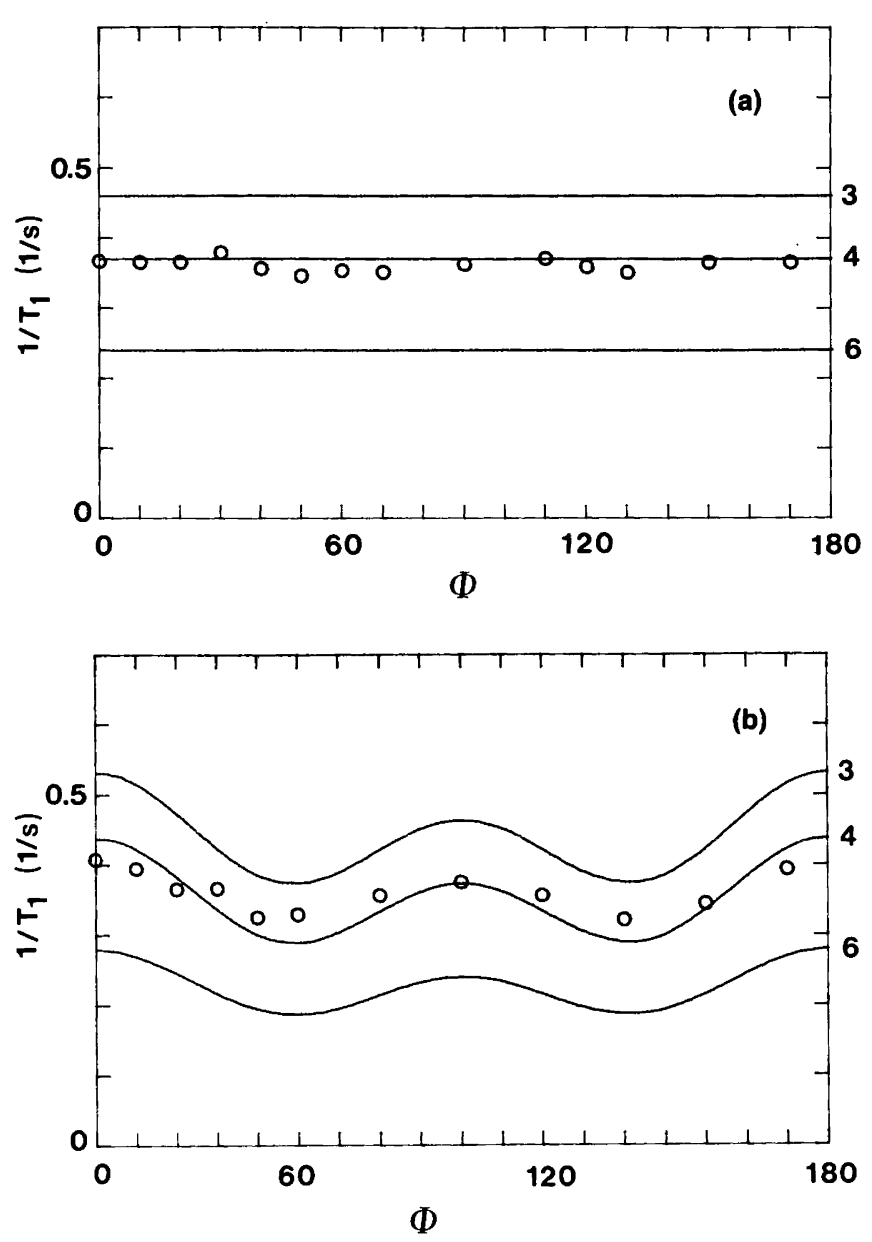

As a complement to the measurements of $T_{1}$, we have measured the spin-lattice relaxation times in the rotating frame $\left(T_{10}\right)$ and the dipolar relaxation time $\left(T_{1 \mathrm{D}}\right)$ for the powdered sample as a function of temperature between 255 and 375 $\mathrm{K}$. These measurements were made at $v_{0}=91.0$ $\mathrm{MHz}$ and $v_{0}=90.0 \mathrm{MHz}$, respectively. In the $T_{10}$ measurements, the locking field $B_{1}$ was $1.04 \mathrm{mT}$, corresponding to $v_{1}=44 \mathrm{MHz}$. The results of these measurements are shown in Fig. 4.

In addition, the relaxation times $T_{10}$ and $T_{1 \mathrm{D}}$ have been measured for the single crystals as a function of orientation, $T_{10}$ for a rotation about axis 2 and $T_{1 \mathrm{D}}$ for a rotation about axis 1. For $T_{1 \varrho}$, $v_{0}$ was $91 \mathrm{MHz}$ and $B_{1}$ was $1.32 \mathrm{mT}\left(v_{1}=56\right.$ $\mathrm{KHz}$ ). For $T_{1 \mathrm{D}}, v_{0}$ was $90 \mathrm{MHz}$. The temperatures during the measurements of the orientation dependences of $T_{1 \mathrm{e}}$ and $T_{1 \mathrm{D}}$ were $291 \mathrm{~K}$ and $313 \mathrm{~K}$, respectively. The results of these measurements are shown in Fig. 5.

\section{Analysis of the relaxation data}

In order to identify the principal origins of the measured relaxation rates, theoretical calculations of the latter were made on the basis of different diffusive models including both rotational and translational motions of $\mathrm{H}_{2} \mathrm{O}$ molecules and $\mathrm{NO}_{2}{ }^{-}$ions. Such diffusive motions must fulfill certain conditions in order to affect the relaxation of the protons and yet be consistent with the known structural data:

1. Motions of at least some nuclei with spin $\neq 0$, i.e. ${ }^{14} \mathrm{~N},{ }^{1} \mathrm{H},{ }^{135} \mathrm{Ba},{ }^{137} \mathrm{Ba}$ or possibly the lowabundant ${ }^{17} \mathrm{O}$ in the present case, must be involved. 


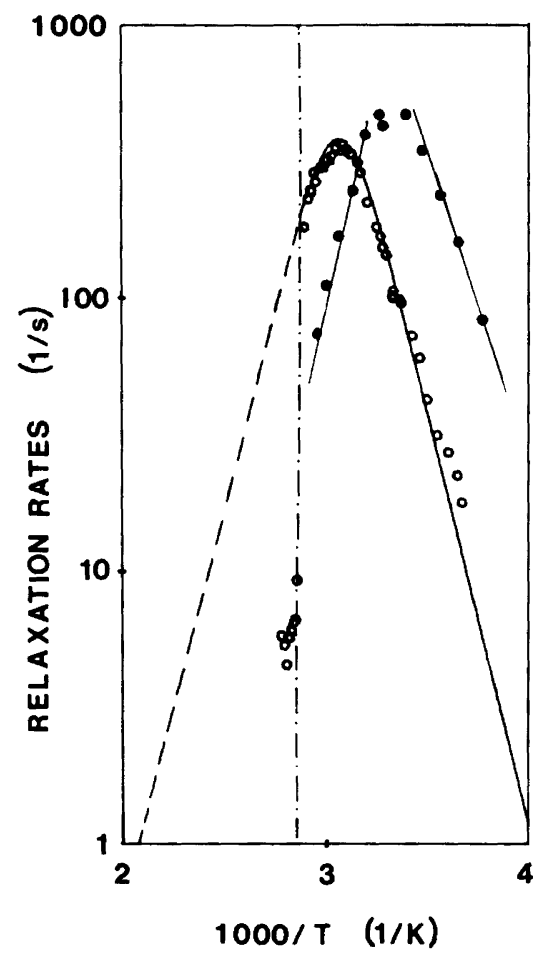

Fig. 4. Temperature dependence of the proton relaxation rates $\left(T_{1 \mathrm{e}}^{-1}\right.$ and $\left.T_{10}^{-1}\right)$ in polycrystalline $\mathrm{Ba}\left(\mathrm{NO}_{2}\right)_{2} \cdot \mathrm{H}_{2} \mathrm{O}$. The experimental data are illustrated by open circles for $T_{10}{ }^{-1}$ at $v_{0}=91 \mathrm{MHz}, v_{1}=44$ $\mathrm{kHz}$, and by filled circles for $T_{10}^{-1}$ at $v_{0}=90 \mathrm{MHz}$. The calculated rates for the final dynamical model (model 1) are also indicated by solid lines.

2. Since distinct positions of the atoms are observed in the structure determined by diffraction, the only possible type of motion is "hopping", i.e. the atoms spend most of the time in the potential wells corresponding to equilibrium positions and only a very small fraction of the time moving between these potential wells.

3. Since there is no evidence of disorder of the atoms in the room temperature phase from the diffraction data, only motions in which a pair of nuclei (or a larger group of nuclei) of the same kind exchange sites need to be considered.

On the basis of these considerations the following models were investigated:

1. A $180^{\circ}$ flip motion of the water molecule about its two-fold axis.
2. Translational diffusion of the water molecule.

3. Translational diffusion of both crystallographically independent nitrite ions.

4. Translational diffusion of the nitrite ion hydrogen-bonded to two water molecules.

5. Translational diffusion of the other independent nitrite ion.

6. Exchange of a pair of $\mathrm{NO}_{2}^{-}$ions in two neighbouring sites.

7. $180^{\circ}$ flip motion of the nitrite ions about their 2-fold axes.

8. Translational diffusion of barium ions.

Relaxation rates of different dynamical processes are furthermore assumed to be additive.

In addition to the above processes involving the motion of the constituent molecules and ionic species in $\mathrm{Ba}\left(\mathrm{NO}_{2}\right)_{2} \cdot \mathrm{H}_{2} \mathrm{O}$, paramagnetic impurities might also influence the relaxation of the protons. The effect of this is hard to estimate; however recrystallisation of the sample led to no appreciable change in the relaxation times, which may be taken as an indication that impurities had no major effect on the relaxation times for this sample in the temperature ranges studied.

In the calculations, the motions were assumed to be characterized by a correlation time $\tau$. The correlation time was assumed to follow the Arrhenius relation

$$
\tau=\tau_{0} \mathrm{e}^{E / R T}
$$

In the analysis of the laboratory frame and the rotating frame spin-lattice relaxation data, the following relations were used for the contributions of like spins to the relaxation rates:

$$
\begin{aligned}
& T_{1}^{-1}=\frac{2}{3} K^{(1)} \frac{\tau}{1+\omega_{0}{ }^{2} \tau^{2}}+\frac{8}{3} K^{(2)} \frac{\tau}{1+4 \omega_{0}{ }^{2} \tau^{2}} \\
& T_{10}{ }^{-1}=K^{(0)} \frac{\tau}{1+4 \omega_{1}{ }^{2} \tau^{2}}+\frac{5}{3} K^{(1)} \frac{\tau}{1+\omega_{0}{ }^{2} \tau^{2}} \\
& +\frac{2}{3} K^{(2)} \frac{\tau}{1+4 \omega_{0}{ }^{2} \tau^{2}}
\end{aligned}
$$

The equations were modified appropriately for contributions from unlike spins. ${ }^{8}$

Here, $\omega_{0}=2 \pi v_{0}, \omega_{1}=2 \pi v_{1}$ and the relaxation constants $K^{(0)}, K^{(1)}$ and $K^{(2)}$ were calculated from the second moment tensors in the presence and in the absence of the motion causing the relaxation. ${ }^{9.10}$ For the calculations of second moment 
Fig. 5. Calculated (solid line) and experimental (circles) proton $T_{10}^{-1}$ (a) and $T_{10}^{-1}(\mathrm{~b})$ for a single crystal of $\mathrm{Ba}\left(\mathrm{NO}_{2}\right)_{2} \cdot \mathrm{H}_{2} \mathrm{O}$ as a function of orientation. The calculated rates correspond to 291 and $313 \mathrm{~K}$, respectively. For $T_{10}^{-1}, v_{0}$ was $91 \mathrm{MHz}$ and $v_{1}=56 \mathrm{kHz}$. For $T_{10}^{-1}, v_{0}$ was 90 $\mathrm{MHz}$.
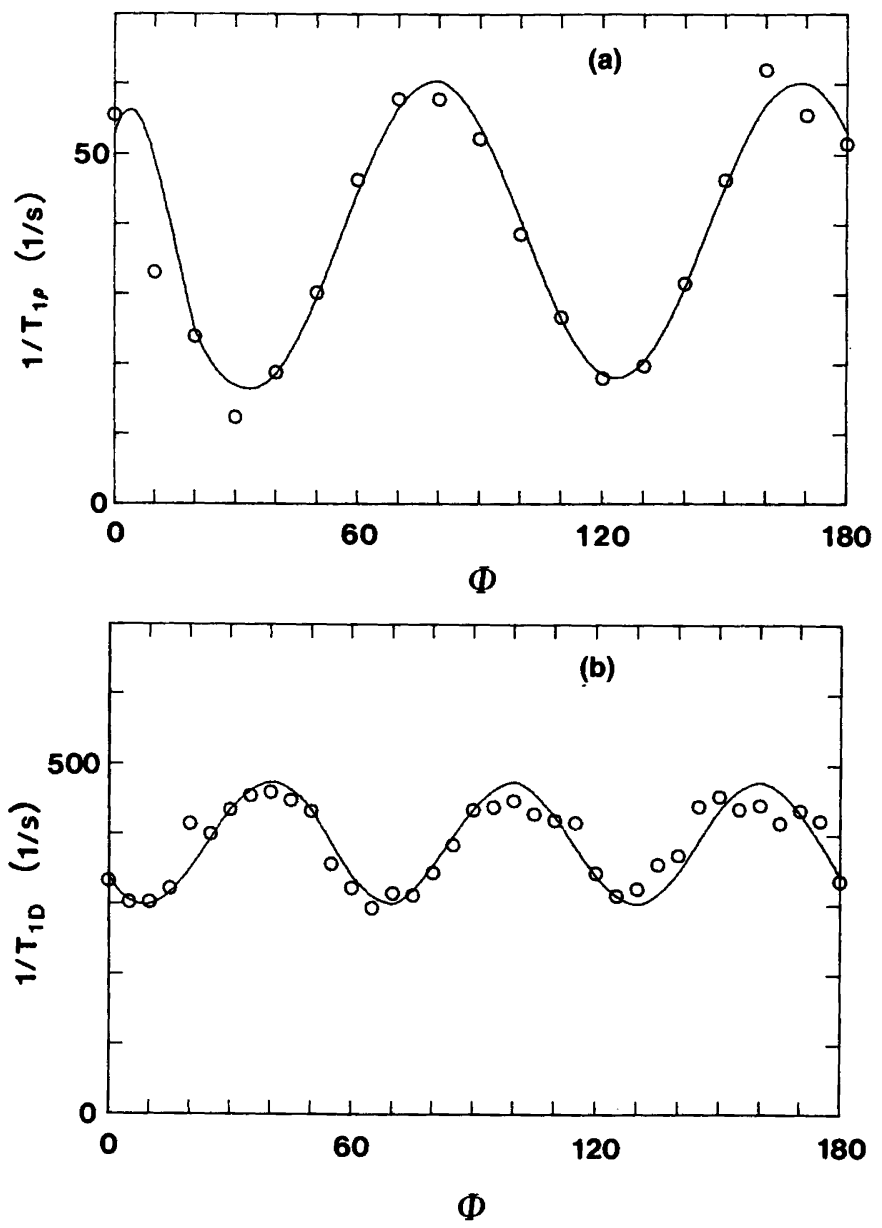

tensors, the positional parameters for all nuclei were taken from the neutron diffraction study. ${ }^{5}$ For powder samples, the relaxation constants $K^{(0)}, K^{(1)}$ and $K^{(2)}$ are identical. The procedure of Chiba and Miyajima ${ }^{11}$ was used for the inclusion of the effect of natural abundance ${ }^{17} \mathrm{O}$ on the proton relaxation rates.

For calculations of the theoretical dipolar relaxation rate $T_{1 \mathrm{D}}{ }^{-1}$, two different approaches were used, depending on the relative magnitude of $\tau^{-1}$ and $\omega_{10 c}$ (the precession frequency of the protons in their local magnetic field). For $\tau \ll 1 / \omega_{\mathrm{loc}}$, the relaxation rate $T_{1 \mathrm{D}}^{-1}$ may be written as

$T_{1 \mathrm{D}}^{-1}=C_{1} \tau+C_{2} \frac{\tau}{1+\omega_{\mathrm{o}}{ }^{2} \tau^{2}}+C_{3} \frac{\tau}{1+4 \omega_{\mathrm{o}}{ }^{2} \tau^{2}}$ where $C_{1}, C_{2}$ and $C_{3}$ are constants of the same order containing the strength of the dipolar interactions. ${ }^{12}$ For correlation times much longer than $1 / \omega_{0}$, the first term in (3) is the only significant term.

For very slow molecular motions, i.e. when $\tau \gg 1 / \omega_{\text {loc }}$, the strong collision theory of Slichter and Ailion ${ }^{13}$ was used.

\section{Results}

Temperature dependence of relaxation rates. Near $350 \mathrm{~K}$, there appears to be an abrupt change in the relaxation rates, by a factor of about 10 . This temperature corresponds to the phase transition reported earlier. ${ }^{6,7}$ However, since the sample undergoes a partial dehydration ${ }^{6}$ at and above this temperature, this result is difficult to analyze 
properly. In the low temperature phase below $350 \mathrm{~K}$, it is clear that at least two different processes determine $T_{1}$ (Fig. 2), and $T_{10}$ and $T_{1 \mathrm{D}}$ (Fig. 4). One process, associated with an activation energy of $57 \pm 3 \mathrm{~kJ} \mathrm{~mol}^{-1}$, dominates $T_{1 \mathrm{e}}$ and $T_{1 \mathrm{D}}$ in this region, and another process with $E=25 \pm 1 \mathrm{~kJ} \mathrm{~mol}^{-1}$ dominates $T_{1}$. In the low temperature phase, between the phase transition temperature and the relaxation rate maximum, $T_{1}$ shows an indication of an additional relaxation mechanism; this is discussed further below.

The experimental relaxation rates $T_{10}^{-1}$ and $T_{\mathrm{ID}}^{-1}$ (Fig. 4) are reproduced very well theoretically using model $1\left(\mathrm{H}_{2} \mathrm{O}\right.$ flip motion $)$. The calculated temperature dependence of the relaxation rates involve only two adjustable parameters for each dynamical process, viz. the activation energy $E$ and the pre-exponential factor $\tau_{0}$ in the Arrhenius relation, whereas the calculated maximum relaxation rate is determined by which of the five dynamical models that is used. The fitted value of $\tau_{0}$ for the $\mathrm{H}_{2} \mathrm{O}$ flip model is $1 \times 10^{-15} \mathrm{~s}$, and the corresponding activation energy is $57 \mathrm{~kJ}$ $\mathrm{mol}^{-1}$. The observed maximum relaxation rate for $T_{1 \mathrm{e}}$ is $3.7 \times 10^{2} \mathrm{~s}^{-1}$. For the $\mathrm{H}_{2} \mathrm{O}$ flip motion, this maximum relaxation rate, calculated using (2), is $3.8 \times 10^{2} \mathrm{~s}^{-1}$, and for model $2\left(\mathrm{H}_{2} \mathrm{O}\right.$ diffusion) is $2.8 \times 10^{5} \mathrm{~s}^{-1}$. For the other models, the calculated maximum relaxation rates are all $<19 \mathrm{~s}^{-1}$.

Fig. 2 shows the $T_{1}$ measurements together with theoretical calculations of the maximum relaxation rates based on the eight different models described above. Model 3 (translational diffusion of both $\mathrm{NO}_{2}^{-}$ions) comes closest to reproducing the experimental maximum relaxation rate for the room temperature phase. The magnitude of the theoretical maximum relaxation rate obtained using model 4 is about $94 \%$ of that obtained using model 3 . It is clear that of the models investigated, only models 3,4 and 6 are reasonably close to reproducing the experimental data, with a slight preference for model 3 . The fitted value of $\tau_{0}$ for this model is $6 \times 10^{-14} \mathrm{~s}$, and the corresponding activation energy $25 \mathrm{~kJ} \mathrm{~mol}^{-1}$. The deviation to higher relaxation rates for the lowtemperature phase close to the phase transition is not fully understood. It might, in part, be a result of the $\mathrm{H}_{2} \mathrm{O}$ flip motion becoming a significant relaxation mechanism.

Orientational dependence of relaxation rates. The orientational dependences of the relaxation rates often provide additional evidence to assist in the identification of the molecular motions responsible for the relaxation. With the present experimental set-up, there is a considerably larger uncertainty in the temperature measurements for single crystals than for polycrystalline samples. The absolute magnitudes of the measured relaxation times for single crystals are thus less significant; more important is the relative variation in the relaxation rate with crystal orientation.

For $T_{1}$, only a very small orientational dependence could be observed. The results are shown in Fig. 3, together with theoretically calculated relaxation rates based on models 3,4 and 6 . The orientational dependence of $T_{1}$ is not only small but also very similar for models 3,4 and 6 . We therefore cannot distinguish between these three models on the basis of the variation in relaxation rate with crystal orientation.

It should be noted that for the measurements shown in Fig. 3, the uncertainty in the temperature is quite unimportant, since a temperature difference of, say, $1^{\circ}$ close to the relaxation rate maximum will cause only a negligible change in $T_{1}^{-1}$ (about $1 \%$ ).

For $T_{1 \mathrm{e}}$ and $T_{1 \mathrm{D}}$, considerably larger variations in the relaxation rates with the orientation were observed. The results are shown in Fig. 5, together with theoretically calculated relaxation rates based on the $\mathrm{H}_{2} \mathrm{O}$ flip model. These results provide additional strong support for the conclusion that the $\mathrm{H}_{2} \mathrm{O}$ flip motion is the major relaxation source contributing to $T_{1 \mathrm{e}}$ and $T_{1 \mathrm{D}}$ in the temperature range studied.

\section{Summary and discussion}

By measuring ${ }^{1} \mathrm{H}$ relaxation rates as a function of temperature in polycrystalline samples of $\mathrm{Ba}\left(\mathrm{NO}_{2}\right)_{2} \cdot \mathrm{H}_{2} \mathrm{O}$, at least two different dynamical processes have been observed. For the room temperature phase, one of these processes is clearly the common $180^{\circ}$ flip motion of the $\mathrm{H}_{2} \mathrm{O}$ molecules. The flip rate for this motion, expressed as the inverse of the correlation time derived from the relaxation data, is shown in Fig. 6 . The motion is characterized by an activation energy of 57 $\mathrm{kJ} \mathrm{mol}^{-1}$; this is in the upper half of the range observed for other solid hydrates. The associated pre-exponential factor, $\tau_{0}$, of the Arrhenius relation for the correlation time of this motion is 


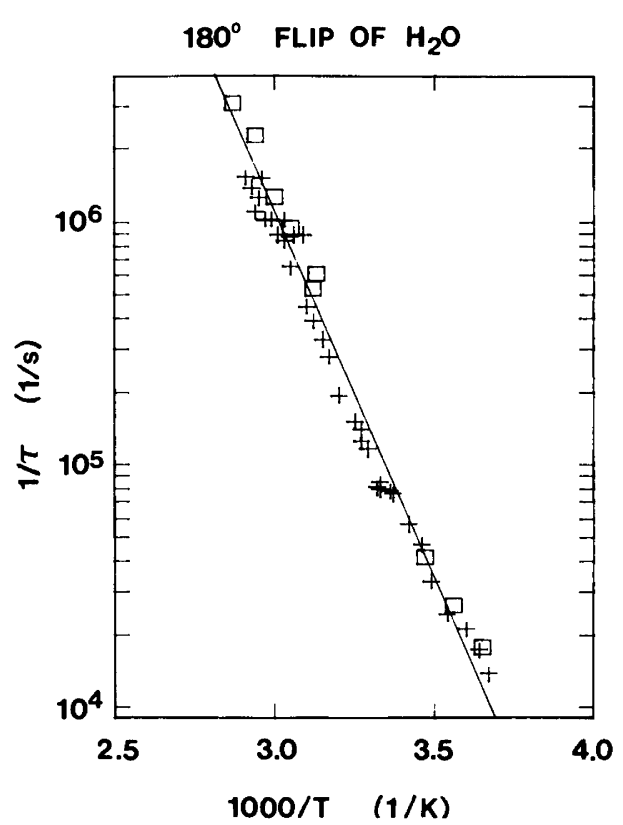

Fig. 6. Temperature dependences of the inverse correlation times for $180^{\circ}$ flip motion of the $\mathrm{H}_{2} \mathrm{O}$ molecules. The experimental data are illustrated by + and $\square$, obtained from relaxation measurements of the temperature dependences of $T_{1 \mathrm{e}}$ and $T_{1 \mathrm{D}}$, respectively. The calculated inverse correlation times for the final dynamical model are indicated by a solid line.

$1 \times 10^{-15}$ s. Slotfeldt-Ellingsen and Pedersen ${ }^{2}$ have discussed the pre-exponential factor for flipping $\mathrm{H}_{2} \mathrm{O}$ molecules in terms of dynamical rate theory as formulated by Feit. ${ }^{14}$ Using their relation between the activation energy and the preexponential factor, we calculate a $\tau_{0}$ value of $1 \times 10^{-16} \mathrm{~s}$ for $E=57 \mathrm{~kJ} \mathrm{~mol}^{-1}$, i.e. one-tenth of our observed value. This is, however, well within the scatter observed for measured $\tau_{0}$ values about the value predicted by the relation in Ref. 2 . The deviation in the present case is not unreasonably large, considering the fact that $\tau_{0}$ varies by almost 6 orders of magnitude when $E$ changes from 10 to $70 \mathrm{~kJ} \mathrm{~mol}^{-1}$ (roughly the range of values observed for solid hydrates). In Ref. 2 , it is suggested that the deviations of the measured $\tau_{0}$ values from the predicted values is most probably due to differences in activation entropy for the flip motion in different hydrates: in $\mathrm{Ba}\left(\mathrm{NO}_{2}\right)_{2} \cdot \mathrm{H}_{2} \mathrm{O}$, a lowering of the activation entropy by about $30 \%$ would account for the discrepancy in $\tau_{0}$.
NMR STUDY OF Ba( $\left(\mathrm{NO}_{2}\right)_{2} \cdot \mathrm{H}_{2} \mathrm{O}$

For the second process in the room temperature phase, our data suggest either an exchange of $\mathrm{NO}_{2}^{-}$between neighbouring sites, or a translational diffusion of the $\mathrm{NO}_{2}{ }^{-}$ions. This interpretation is strongly supported by the ${ }^{14} \mathrm{~N} N Q R$ data reported by Oja, Marino and Bray. ${ }^{15}$ In studying the temperature dependence of the four spectral lines observed, they found that one pair of lines (corresponding to one of the two non-equivalent $\mathrm{NO}_{2}{ }^{-}$ions) disappears at temperatures above 172-175 K. The second pair of lines (corresponding to the other $\mathrm{NO}_{2}{ }^{-}$ion) shows the same behaviour at temperatures around $300 \mathrm{~K}$. This would occur if the quadrupole coupling of the nitrogen nuclei were modulated by suitable random fluctuations of a sufficiently high frequency. Oja et al. suggest that these fluctuations are caused by the onset of reorientations of either the $\mathrm{H}_{2} \mathrm{O}$ molecules or the $\mathrm{NO}_{2}^{-}$ions. It is certainly clear that the $\mathrm{H}_{2} \mathrm{O}$ molecules reorient; however, the $180^{\circ}$ flip motion of the $\mathrm{H}_{2} \mathrm{O}$ molecules would hardly affect the ${ }^{14} \mathrm{~N}$ NQR signal. For the other possibility, i.e. diffusive motion of the $\mathrm{NO}_{2}^{-}$ions, there are essentially two main alternatives:

1. The $\mathrm{NO}_{2}^{-}$ions reorient without leaving their sites: as discussed above, the only type of reorientation compatible with structural data would then be a $180^{\circ}$ flip motion analogous to the $\mathrm{H}_{2} \mathrm{O}$ flip motion. However, the quadrupole coupling of the ${ }^{14} \mathrm{~N}$ nuclei is invariant to such motions. Reorientation of this type cannot, therefore, explain the disappearance of the ${ }^{14} \mathrm{~N}$ NQR signal.

2. Exchange between neighbouring $\mathrm{NO}_{2}^{-}$ions (possibly extended to translational diffusion through continued exchange with next neighbours). Because of the symmetry of the crystal, an exchange of sites even between two equivalent $\mathrm{NO}_{2}^{-}$ions would, in general, rotate the electric field gradient felt by the nitrogen nuclei. The motion would, therefore, produce a time-dependent modulation of the ${ }^{14} \mathrm{~N}$ quadrupole coupling, which could cause a disappearance of the NQR signal as observed. This alternative is also consistent with our relaxation data. The results in Ref. 15 clearly indicate a difference between the rate of motion of the two non-equivalent $\mathrm{NO}_{2}^{-}$ions, although we cannot resolve any such difference. The motion is characterized by an activation energy of $25 \mathrm{~kJ} \mathrm{~mol}^{-1}$ (average for the two non-equivalent $\mathrm{NO}_{2}^{-}$ions). If one assumes 
that the motion is a transational diffusion, a pre-exponential factor of $1.1 \times 10^{-13} \mathrm{~s}$ can be calculated. From the correlation time $\tau$, one can then also estimate, albeit very crudely, a diffusion coefficient $D$ : Assuming that the elementary diffusion step is a jump of the $\mathrm{NO}_{2}{ }^{-}$ ions between equivalent sites a distance $d$ apart, one obtains

$$
D=\frac{d^{2}}{6 \tau}
$$

valid for three dimensional diffusion. Using $d$ $=3.5 \AA$, (4) gives an estimated diffusion coefficient of $9 \times 10^{-8} \mathrm{~cm}^{2} \mathrm{~s}^{-1} \mathrm{~s}$ at $300 \mathrm{~K}$, decreasing to $3 \times 10^{-9} \mathrm{~cm}^{2} \mathrm{~s}^{-1}$ at $225 \mathrm{~K}$.

The above values are very high diffusion rates, much higher than observed for molecules in other solids even in extreme cases. ${ }^{16}$ It is therefore very hard to believe that an interpretation in terms of translational diffusion of $\mathrm{NO}_{2}^{-}$ions can be correct. The other alternative, involving pairwise exchange of $\mathrm{NO}_{2}{ }^{-}$ions, agrees less well with the observed relaxation rates, although the discrepancy is not unreasonably large. In addition, there is the complication of relaxation induced by paramagnetic impurities. We cannot, therefore, unequivocally determine the process responsible for the spin-lattice relaxation $\left(T_{1}\right)$ in the room temperature phase.

Acknowledgements. We thank H. Karlsson for invaluable assistance in orienting the sample crystals. This work has been supported by grants from the Swedish Natural Science Research Council, which are hereby gratefully acknowledged.

\section{References}

1. Larsson, K. and Tegenfeldt, J. J. Magn. Reson. 64 (1985) 451

2. Slotfeldt-Ellingsen, D. and Pedersen, B. J. Phys. Chem. Solids 38 (1976) 65.

3. Liminga, R., Abrahams, S. C. and Bernstein, J. L. J. Appl. Crystallogr. 13 (1980) 516.

4. Abrahams, S. C., Bernstein, J. L. and Liminga, R. J. Chem. Phys. 72 (1980) 5857.

5. Kvick, А̊., Liminga, R. and Abrahams, S. C. J. Chem. Phys. 76 (1982) 5508.

6. Gallagher, P. K., Abrahams, S. C., Wood, D. L., Schrey, F. and Liminga, R. J. Chem. Phys. 75 (1981) 1903.

7. Wood, D. L. J. Chem. Phys. 75 (1981) 4809.

8. Wolf, D. Spin-temperature and Nuclear-spin Relaxation in Matter, Oxford University Press, Oxford 1979.

9. Sjöblom, R. J. Magn. Reson. 22 (1976) 425.

10. Tegenfeldt, J. and Sjöblom, R. J. Magn. Reson. 55 (1983) 372.

11. Chiba, T. and Miyajima, S. J. Chem. Phys. 83 (1985) 6385.

12. Van Steenwinkel, R. Z. Naturforsch., Teil A24 (1969) 1526.

13. Slichter, C. P. and Ailion, D. Phys. Rev. A 135 (1964) 1099.

14. Feit, M. D. Phys. Rev. B 5 (1972) 2145.

15. Oja, T., Marino, R. A. and Bray, P. J. Phys. Lett. A 28 (1968) 16.

16. Chezeau, J. M. and Strange, J. H. Phys. Reports 53 (1979) 1.

Received July 16, 1986. 